\title{
Temperature Behavior of the Electrical Conductivity of
}

\section{Emim-Based Ionic Liquids in Liquid and Solid States}

\author{
J. Vila, ${ }^{a}$ C. Franjo, ${ }^{a}$ J.M. Pico, ${ }^{a}$ L.M. Varela, ${ }^{b}$ O. Cabeza ${ }^{*}$, \\ ${ }^{a}$ Dpto. de Física, Fac. de Ciencias, Universidade da Coruña. Campus da Zapateira s/n, \\ 15072 A Coruña, SPAIN \\ ${ }^{b}$ Grupo de Nanomateriales y Materia Blanda. Dpto. de Física de la Materia Condensada. Fac. \\ de Física, Universidad de Santiago de Compostela. E-15782. Santiago de Compostela. SPAIN
}

\begin{abstract}
In this paper we present experimental measurements of the temperature dependence of the electrical conductivity, $\sigma$, in four ionic liquid compounds (ILs) in both the liquid and solid states and at atmospheric pressure. The chemicals measured are composed by the 1-ethyl-3-methyl-imidazolium $\left(\mathrm{EMIM}^{+}\right)$cation, which has been combined with four different anions: $\mathrm{Cl}^{-}, \mathrm{Br}^{-}, \mathrm{BF}_{4}{ }^{-}$and ethyl sulfate $\left(\mathrm{ES}^{-}\right)$. In the liquid state, the temperature dependence of $\sigma$, for the four ILs follows the Vogel-Tamman-Fulcher (VTF) equation with high precision. Around the transition between the solid and liquid states, the electrical conductivity of the three ILs with lighter anions presents an hysteresis loop, that can be explained as the apparition of supercooled liquid. In contrast, the EMIM-ES presents a smooth transition, without any jump in the $\sigma$, value or any hysteresis loop (probably because its melting point is reported to be below the minimum temperature measured by us). Finally, the jump in $\sigma$, is not related with the glass transition because its temperature value is well below the minimum temperature measured, and at that glass transition temperature the $\sigma$, value is below the resolution of our conductivity meter $(2 \mathrm{nS} / \mathrm{cm})$.
\end{abstract}

Keywords: ionic liquids, electrical conductivity, temperature, phase transition, hysteresis.

\section{Introduction}

Room temperature ionic liquids (RTILs or simply ILs) are solvent-free ionic fluids composed solely by ions having melting points around room temperature, the highest acceptable melting temperature is about $373 \mathrm{~K}$. These compounds

\footnotetext{
* Corresponding author. E-mail address: oscabe@udc.es
} 
have very interesting properties as low vapor pressure, high electrical conductivity, chemical stability, broad range of temperatures for liquid state, broad electrochemical window. As a consequence of the above mentioned properties there were many proposed applications for ILs in the field of green chemistry, among which we can name those of replacement of organic solvents, as catalytical medium, charge transport in energy generators, batteries or electrolytic medium in electrodeposition processes [1-3]. In fact there are important advances in the use of ionic liquids in Li batteries [4], as electrolyte [5], and even in solar power applications [6]. To develop these applications it is first necessary to know the value of the physical magnitudes of these liquids, and their behavior with temperature. Also the physical data are necessary to develop a theoretical model to explain and to predict them in different compounds (which doesn't exist nowadays), and to improve the molecular dynamics simulations now in progress [7-9]. Despite it is well known since early studies of transport processes in ILs that many of these systems are often good glass formers [10-14], so they can be cooled down avoiding crystallization, the particular details of the glasses formed in these ionic systems are still far from being well characterized [11]. Moreover, most of the presently known features about the physics of supercooled ionic liquids have been discovered in a highly empirical fashion. The character of glass-former means that below the melting point, $T_{m}$, the supercooled material retains its liquid character falling out of equilibrium down to it, when the glass temperature, $T_{g}$, is reached. In other words, $T_{g}$ is the temperature until which the configurational entropy, the essential characteristic of a liquid, does not vanish. Good glass formers are systems with low crystal nucleation probabilities at all temperatures down to $T_{g}$. This temperature is of the utmost importance for a proper understanding of the transport properties of ILs at room temperature and, therefore, an adequate characterization of the glass transition presents a deep theoretical and applied interest [14].

\section{Experimental details}

Here, we present experimental measurements of the electrical conductivity versus temperature at atmospheric pressure. The range of temperatures covered in this paper depends on each IL, and it extends from $200 \mathrm{~K}$ to $500 \mathrm{~K}$. The measured ILs can be classified in two different kinds. The first one includes 1ethyl-3-methyl imidazolium chloride (EMIM-Cl) and EMIM-Br. They were purchased from Aldrich, and have a purity $>97 \%$ for EMIM-Br, and $>93 \%$ (BASF quality) for EMIM-Cl. These two compounds are solid at room temperature and they are hygroscopic, so they have been manipulated and measured in Ar atmosphere. The second group of ILs include EMIM-BF 4 and EMIM-ES (commercially known as ECOENG ${ }^{\mathrm{TM}} 212$ ). They were purchased to Solvent Innovations and both present purities better than 98\%. These two compounds are liquid at room temperature and are inert to air (non hygroscopic), so they have been manipulated and measured without any special atmosphere. All the four ILs measured have a low viscosity and high electrical conductivity, 
and so they are indicated for electrical applications [1-3]. Moreover, they are relatively cheap and some of them produced in semi-industrial scale.

The electrical conductivity data $(\sigma)$ we present here have been measured using a CRISON GLP31 conductivimeter with a resolution of around 1\% (or $2 \mathrm{nS} / \mathrm{cm}$ ), which uses an ac current with a frequency of $500 \mathrm{~Hz}$. All data presented here for each compound have been measured several times in different samples (and different days) to ensure their reproducibility within $95 \%$ in absolute value. The sample is immersed in a thermostated bath, and its temperature is increased up the maximum chosen for each sample (and so we melt the two ILs that are solid at room temperature). From the maximum temperature the sample is cooled step by step (of about $5 \mathrm{~K}$ out the transition, and smaller around it) and the $\sigma$ value is measured only when the sample temperature is stable. Below $260 \mathrm{~K}$ we cool the sample with liquid nitrogen. When the $\sigma$ value is about the resolution of the conductivity meter the temperature is again increased step by step, and the measurement is also isothermal. The general procedure has been described in more detail previously $[12,15]$.

\section{Theoretical discussion}

The essential and universal features of the thermodynamics and kinetics of glassy systems are the Kauzmann paradox on the entropy crisis [16] and the VogelTamman-Fulcher (VTF) law for the temperature dependence of the relaxation and transport processes [17-19]. The relation between the melting point temperature, $\mathrm{T}_{\mathrm{m}}$, and the glass transition temperature is usually well represented by the so called " $2 / 3$ " rule for glass-formers $\left(T_{g} / T_{m}=2 / 3\right)$ [20]. Kauzmann paradox -the fact that a supercooled liquid may have a negative configuration entropy below the glass transition- was solved by Gibbs and Di Marzio [21], who demonstrated in a statistical-mechanical quasilattice theory the existence of a second-order transition at the temperature $T_{g}$ where the configurational entropy vanishes, remaining zero at lower temperatures.

In this formalism, the transport processes take place by hoppings from sites to vacancies, corresponding to jumps between successive valleys in the multidimensional configuration space. In Einstein's fluctuation theory, the hopping probability is proportional to the configuration number, so this probability is given by [22]:

$$
J=\exp \left(\frac{z S_{K}}{N k_{B}}\right)=\exp \left(-\frac{E}{k_{B}\left(T-T_{g}\right)}\right)
$$

where $E=z N_{0} T_{g} / 2 N$ and $z$ is a number of the order of the number of neighbor particles. Obviously, the above equation is of the VTF type, manifesting the glass-transition crisis in the configurational entropy.

In what the kinetic behaviour of the system is concerned, the glass transition in the low temperature region is associated to an extraordinary slow down of the relaxation and transport (viscosity and diffusion) mechanisms [10,23]. This slow 
down is itself related to the "death of configurations", and enormous reduction of the number of accessible microstates (configurational entropy) that takes place as the temperature falls. As a consequence, the relaxation and transport processes suffer considerable restrictions that are universally governed by the VTF law:

$$
\psi(T)=A \exp \left(-\frac{B}{T-T_{g}}\right)=A \exp \left(-\frac{\widetilde{D} T_{g}}{T-T_{g}}\right)
$$

Traditionally $T_{g}$ has been kinetically defined as the temperature at which the viscosity reaches a certain high value $10^{13} \mathrm{P}$, so it does not exactly coincides with the temperature at which the transport property vanishes exactly (sometimes known as the ideal glass transition temperature [24]), although they are usually so similar that in practice they are identified. On the other hand, $\widetilde{D}$ is inversely proportional to the fragility of the liquid. This property is a measure of the sensitivity of the liquid structure to changes in the temperature. "Fragile" liquids are those in which a subtle variation of the thermal energy provokes the collapse of their glassy structures, changing to states of considerable fluctuation of the structural arrangement and coordination states [25]. These systems comprise liquids characterized by simple non-directional Coulomb interactions or by Van der Waals interactions in substances with many $\pi$ electrons, usually aromatic substances [25]. In these substances, the transport properties show a highly nonArrhenius behavior. The fragile liquids must be opposed to the so called "strong" liquids, that exhibit considerable resistance to internal (structural) rearrangements despite wide variations of temperature, and that can be transformed into fragile liquids by changing their densities. Together with the glass transition temperature, this property of fragility is the other main influence on the fluidity of glass-forming ILs. The incorporation of the advances in glass transition theory into the hole theory of ILs seems obligatory and straightforward, given the evident similitude of the hole theory structural model of ILs and the basic assumptions of the Adam-Gibbs formalism or those of the two-band model. The work in this direction is now in progress.

\section{Results and discussion}

In Fig. 1(a) to (d) we plot the electrical conductivity measured, $\sigma\left(\mathrm{mS} \cdot \mathrm{cm}^{-1}\right)$, of the ILs in logarithmic scale versus temperature, $T(K)$. In the four figures a sketch is focused in the melting region where the electrical conductivity is given in linear scale. The arrows indicate the temperature variation.

As can be observed in Fig. 1, all of the EMIM-based ILs present a similar $\sigma(\mathrm{T})$ exponential dependence in liquid state, which allows that the electrical conductivity value increases more than two orders of magnitude in $200 \mathrm{~K}$ of temperature variation. Following hole theory for transport in molten salts we obtain an Arrhenius type equation for the temperature dependence of the electrical conductivity, which can be written as [22], 


$$
\sigma=\sigma_{\infty} \exp \left[\frac{E_{a}}{k_{B} \cdot T}\right]
$$

where $E_{a}$ is the activation energy for electrical conduction, which indicates the energy needed for an ion to jump to a free hole, $\sigma_{\infty}$ is the maximum electrical conductivity (that it would have at infinite temperature) and $k_{B}$ is the Boltzman constant. Our ILs does not follow the Arrhenius behavior given by Eq. (3). The observed curvature is accounted for by VTF-type equation. As commented in the theoretical section, both Arrhenius and VTF equations are used to explain the temperature behavior of different physical magnitudes, including viscosity, diffusion constant and electrical conductivity. In a recent paper it is given a microscopically explanation for VTF equation for the dielectric relaxation phenomena [26]. This reference is, at our knowledge, the first that gives a theoretical basis for any VTF-type equation. The VTF equation appears in different forms depending of the author [18-20,26-28]. Here we have chosen the most simple and most usual, which reads, similarly to Eq. (2) [27,28]

$$
\sigma=A \cdot \exp \left[-\frac{B}{\left(T-T_{g}\right)}\right]
$$

where A, B and $T_{g}$ are fitting parameters. If we compare Arrhenious equation (3) with VTF equation (4), we can observe that the second is equal to the first if $T_{g}=$ 0 . Thus we can relate the fitting parameters of VTF-type equation with the physical parameters of Arrhenious equation: $A=\sigma_{\infty}$ and $B=E_{a} / k_{B}$. The modified version of the VTF-type equation would read

$$
\sigma=\sigma_{\infty} \exp \left[-\frac{E_{a}}{k_{B} \cdot\left(T-T_{g}\right)}\right]
$$

In Table 1 we show the obtained values of $\sigma_{\infty}, E_{a}$ and $T_{g}$ from the best fit of Eq. (5) to the experimental data, and the corresponding standard deviation, s, defined as usual. It is important to note that, in order to take into account with the same weight all data points, the fits were performed minimizing the following expression:

$$
f=\sum_{i=1}^{N}\left(\frac{\sigma_{c a l}-\sigma_{\exp }}{\sigma_{\exp }}\right)^{2}
$$

where $\sigma_{\text {cal }}$ is the value obtained from Eq. (4), $\sigma_{\exp }$ that measured and $\mathrm{N}$ the number of data points used in the fitting (which is included in Table 1 for each compound). The obtained curves are plotted in Figure 1(a) to (d) for all ILs measured. From the analysis of the three physical parameters obtained summarized in Table 1 we do not observe a trend with the anion size as could be expected. 


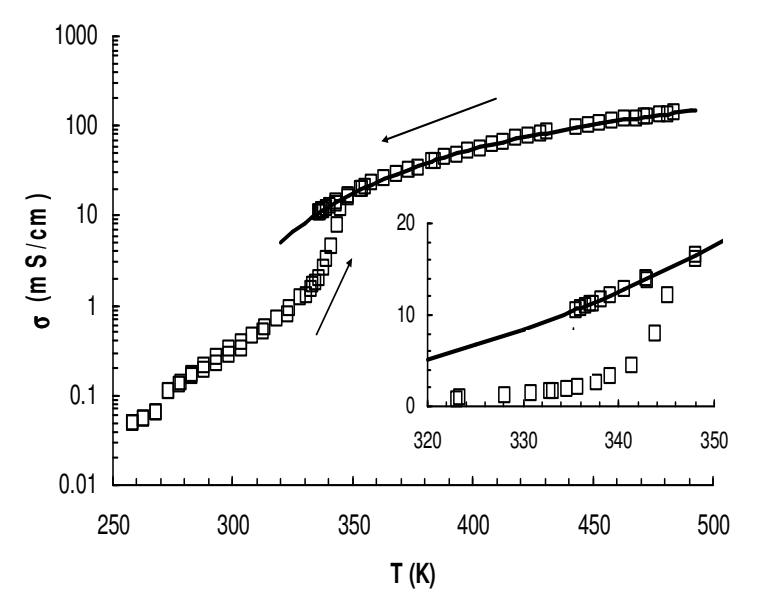

(a)

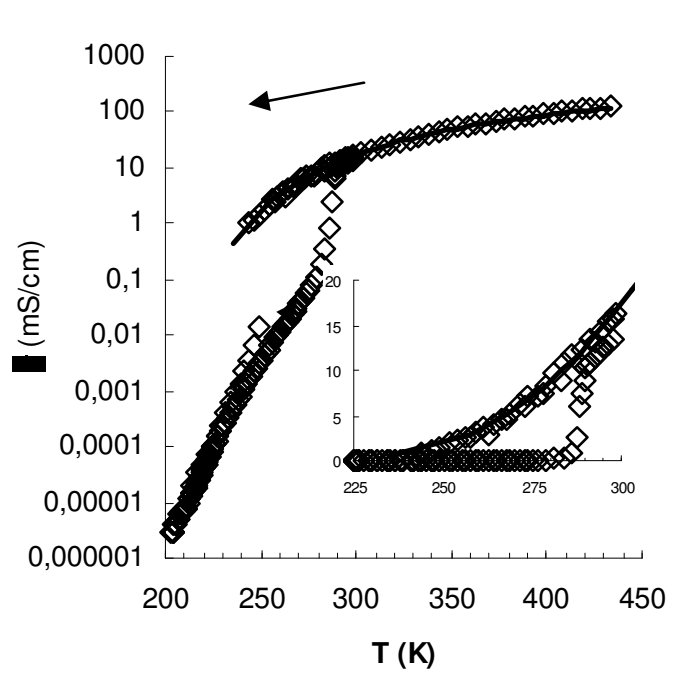

(c)

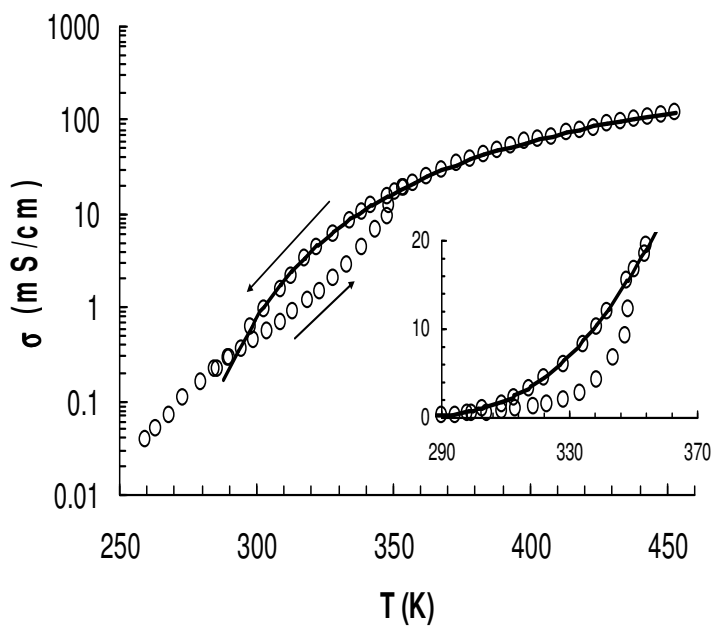

(b)

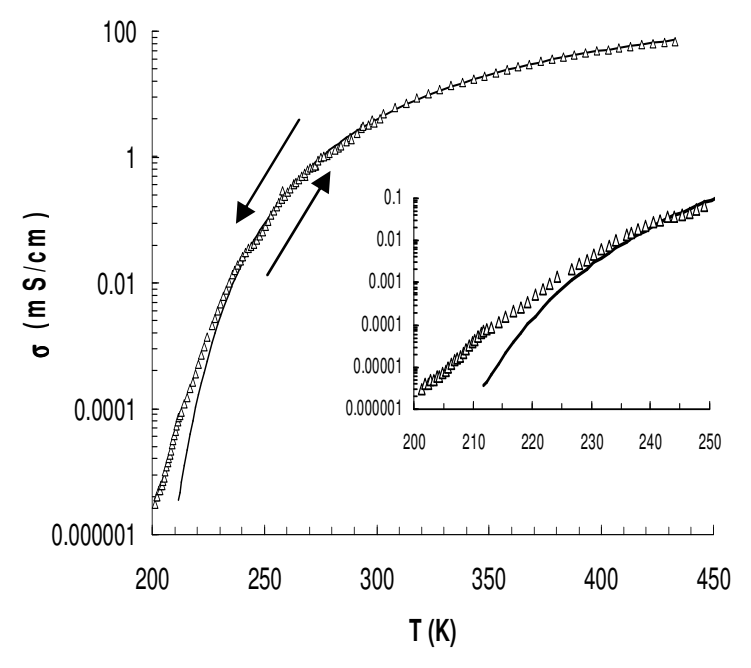

(d)

Figure 1. Semi Arrhenius plot of the electrical conductivity, $\sigma$, vs. temperature for (a) EMIM-Br, (b) EMIM-Cl, (c) EMIM-BF 4 and (d) EMIM-ethyl sulfate. Lines are the best fit of the VTF type Eq. (5) with the parameters given in Table 1. In the inset we show the liquid-solid phase transition in linear scale. Arrows indicate the variation of temperature (cooling or heating).

The most interesting fact observed in our $\sigma(\mathrm{T})$ curves is the apparition of a hysteresis loop at the melting point in three of the compounds (except for EMIMES), although the loop differs among them. A similar phase transition had been observed previously in other ILs $[28,29]$, and it is related with the apparition of 
overcooled liquid below its melting point temperature (obtained from the heating curve). For EMIM-Br in Fig. 1(a), the jump of the $\sigma$ value at the transition, when temperature decreases, is evident in both scales (logarithm and linear), probably because the overcool liquid remains only $10 \mathrm{~K}$. In contrast, for EMIM-Cl in Fig. 1(b) the overcooled liquid remains about $50 \mathrm{~K}$, and so the loop is closed and it doesn't appear any jump there for $\sigma$. For EMIM-BF ${ }_{4}$ in Fig. 1(c) the jump when the sample is cooling is also evident in the logarithm scale (not so clear in linear scale), although the overcooled liquid remains about $50 \mathrm{~K}$. Also in this sample the jump does not get the solid value and this is attained after about $10 \mathrm{~K}$.

Table 1. Theoretical physical magnitudes extracted from the best fit of experimental data to the VTF-type Eq. (5). The number of data points used in the fit, and their standard deviations, s, are included.

\begin{tabular}{lccccc}
\hline & $\sigma_{\infty}\left(\mathrm{mS} \mathrm{cm}^{-1}\right)$ & $E_{a}(\mathrm{meV})$ & $T_{g}(\mathrm{~K})$ & $\mathrm{N}$ & $\sigma\left(\mathrm{mS} \mathrm{cm}^{-1}\right)$ \\
\hline EMIM-Cl & 1215 & 44.3 & 230.2 & 44 & 1.00 \\
EMIM-Br & 1060 & 46.6 & 218.8 & 38 & 0.68 \\
EMIM-BF 4 & 1355 & 57.6 & 148.8 & 27 & 0.56 \\
EMIM-ES & 831 & 52.2 & 186.2 & 33 & 0.36 \\
\hline
\end{tabular}

Finally, for EMIM-ES in Fig. 1(d), no hysteresis loop appears, and the data obtained when cooling match exactly with those when heating. It is possible we have not attained the melting point (reported to be about $190 \mathrm{~K}$ ), but the $\sigma$ value was below $2 \mathrm{nS} / \mathrm{cm}$ at $200 \mathrm{~K}$, and so about the resolution of our apparatus (and using the measurement cell suitable for low conductivity). In any case, the VTF curve does not fit the data for temperatures below $235 \mathrm{~K}$, which seems to indicate that the liquid character of EMIM-ES is lost below that temperature. The observed differences between the two last ILs could be due to different anion sizes (much higher for ethyl sulfate), because the fragility of the liquid is higher for EMIM-ES than for EMIM-BF 4 (obtained qualitatively from the values of $E_{a}$ and $T_{g}$ in Table 1). The variation of the loop for the two halide compounds could be due to the different purities of both compounds (purer bromide than chloride). In any case, a theoretical model to explain the data presented here is still lack.

Finally, note that the theoretical value of $T_{\mathrm{g}}$ obtained from the best fit of Eq. (5) is below the minimum temperature measured for all samples. In any case, we doubt that at so low temperatures of $T_{\mathrm{g}}$ the electrical conductivity data could show any anomaly due to the so low value of this magnitude (even with a better conductivimeter). To observe the glass transition it is useful the DSC technique, which allows us to experimentally measure $T_{g}$ due to the change in the thermal capacity of the compound at that temperature, as observed by other authors [28]. 


\section{Conclusions}

In this paper we present measurements for the first time of the temperature dependence of the electrical conductivity for four different EMIM-based ILs in both liquid and solid states when the sample is cooled and heated. The electrical conductivity dramatically increases with temperature, reaching the $\sigma$ value in liquid state more than 100 times in a temperature increase of about $250 \mathrm{~K}$. The theoretical analysis of the temperature dependence of the electrical conductivity in liquid state indicates that all of them follow a VTF type equation. If we compare this semi phenomenological equation with Arrhenious theory we can extract from the fit of the experimental points some very interesting parameters from the theoretical point of view, as the electrical conductivity limit for $\mathrm{T} \rightarrow \infty$, the activation energy and the glass transition temperature. Finally the loop observed around the liquid to solid phase transition is different for the four samples measured and its apparition is related with the existence of overcooled liquid below the melting point.

\section{Acknowledgement}

J.Vila aknowledge "Xunta de Galicia" for the research contract obtained from the PGIDT05PXIC10305PN research project.

\section{References}

1. R.K. Rogers and K.R. Seddon (Editors), in "Ionic Liquids, Industrial Applications to Green Chemistry”, ACS Symp. Series 818, Am. Chem. Soc., Washington, 2002.

2. F. Endres, "Ionic Liquids: Solvents for the Electrodeposition of Metals and Semiconductors", Chem. Phys. Chem. 3 (2002) 144-154.

3. P. Wasserscheid and T. Welton (Editors), in "Ionic Liquids in Synthesis", Wiley-VCH, Verlag, Weinheim, 2003.

4. M. Ishikawa, T. Sugimoto, M. Kikuta, E. Ishiko and M. Kono, "Pure ionic liquid electrolytes compatible with a graphitized carbon negative electrode in rechargeable lithium-ion batteries", J. Power Sources 162 (2006) 658662.

5. M. Galinski, A. Lewandowski and I. Stepniak, "Ionic liquids as electrolytes", Electrochimica Acta 51 (2006) 5567-5580.

6. M.E. Van Valkenburg, R.L. Vaughn, M. Williams and J.S. Wilkes, "Thermochemistry of ionic liquid heat-transfer fluids", Thermochimica Acta 425 (2005) 181-188.

7. S.U. Lee, J. Jung and Y-K. Han, "Molecular dynamics study of the ionic conductivity of 1-n-butyl-3-methylimidazolium salts as ionic liquids", Chemical Physics Letters 406 (2005) 332-340.

8. C. Rey-Castro and L.F. Vega, "Transport properties of the ionic liquid 1ethyl-3-methylimidazolium chloride from equilibrium molecular dynamics simulation. The effect of temperature", J. Phys. Chem. B 110 (2006) 14426-14435. 
9. C. Rey-Castro, A.L. Tormo and L.F. Vega, "Effect of the flexibility and the anion in the structural and transport properties of ethyl-methylimidazolium ionic liquids", Fluid Phase Equilibria (2006). In press.

10. C.A. Angell, in "Complex Behaviour of Glassy Systems", edited by M. Rubí, C. Pérez-Vicente. Springer, Berlin, 1997.

11. M. Videa, $\mathrm{Wu} \mathrm{Xu,} \mathrm{B.} \mathrm{Geill,} \mathrm{R.} \mathrm{Marzke,} \mathrm{C.A.} \mathrm{Angell,} \mathrm{"High} \mathrm{Li}^{+}$self diffusivity and transport number in novel electrolyte solutions", $J$. Electrochem. Soc. 148 (2001) A1352-A1356.

12. J. Vila, P. Ginés, J.M. Pico, C. Franjo, E. Jiménez, L.M. Varela and O. Cabeza, "Temperature dependence of the electrical conductivity in EMIM based ionic liquids. Evidence of Vogel-Tamman-Fülcher behavior", Fluid Phase Equilibria 242 (2006) 141-146.

13. E. Rilo, J. Vila, P. Ginés, M. Domínguez-Pérez, L. Segade, C. Franjo and O. Cabeza, "Electrical conductivity and viscosity of EMIM-Br and EPYR$\mathrm{Br}+\mathrm{AlBr}_{3}$ ionic liquids and their precursors in aqueous solutions". In EUCHEM 2004 Molten Salts Conference Proceedings (Acta Universitatis Wratislaviensis No 2693, Wroclaw, 2004). Pgs. 325-330.

14. G.F. Reynolds and C.J. Dymek, "Primary and secondary room temperature molten salt electrochemical cells", J. Power Sources 15 (1985) 109-118.

15. M. Prego, E. Rilo, E. Carballo, C. Franjo, E. Jiménez and O. Cabeza, "Electrical conductivity data of alkanols from 273 to $333 \mathrm{~K}$ ", J. Molecular Liquids 102 (2003) 83-91.

16. W. Kauzmann, "The nature of the glassy state and the behavior of liquids at low temperatures", Chem. Rev. 43 (1948) 219-256.

17. H. Vogel, Phys. Z. 22 (1921) 645-646.

18. G. Tamman and W. Hesse, "Die Abhängigkeit der Viscosität von der Temperatur bie unterkühlten Flüssigkeiten", Z. Anorg, Allg. Chem. 156 (1926) 245-257.

19. G.S. Fulcher, "Analysis of recent measurements of the viscosity of glasses", J. Am. Ceram. Soc. 8 (1925) 339-355.

20. C. Alba-Simionesco, J. Fan and C.A. Angell, "Thermodynamic aspects of the glass transition phenomenon. II. Molecular liquids with variable interactions", J. Chem. Phys. 110 (1999) 5262-5272.

21. J.H. Gibbs, in "Modern Aspects of the Vitreous State", Butterworths Scientific Publications Ltd., London, 1960.

22. J.O'M. Bockris and A.K.N. Reddy, in "Modern Electrochemistry", Plenum Press, New York, 1998, Chaps. 4 and 5.

23. T. Kitamura, "Quantum field theory of the liquid-glass transition", Phys. Rep. 383 (2003) 1-94.

24. C.A. Angell and E.J. Sare, "Glass-forming composition regions and glass transition temperatures for aqueous electrolyte solutions", J. Chem. Phys. 52 (1970) 1058-1068.

25. C.A. Angell, "The old problems of glass and the glass transition, and the many new twists", Proc. Natl. Acad. Sci. USA 92 (1995) 6675-6682. 
26. R.R. Nigmatullin, S.I. Osokin and G. Smith, "New approach in the description of dielectric relaxation phenomenon: correct deduction and interpretation of the Vogel-Fulcher-Tamman equation", J. Physics: Condensed Matter 15 (2003) 3481-3503.

27. H. Every, A.G. Bishop, M. Forsyth and D.R. MacFarlane, "Ion diffusion in molten salt mixtures", Electrochimica Acta 45 (2000) 1279-1284.

28. D.R. McFarlane, J. Sun, J. Golding, P. Meakin and M. Forsyth, "High conductivity molten salts based on the imide ion", Electrochimica Acta 45 (2000) 1271-1278.

29. K. Ito, N. Nishina and H. Ohno, "Enhanced ion conduction in imidazolium-type molten salts", Electrochimica Acta 45 (2000) 12951298. 